\title{
Ankle brachial index (ABI) in a cohort of older women in the Philippines: Prevalence of peripheral artery disease and predictors of $\mathrm{ABI}$
}

\author{
Christopher W. Kuzawa ${ }^{1,2}$ (1) | Tyler M. Barrett ${ }^{1}$ | Judith B. Borja ${ }^{3,4}$ | Nanette R. Lee I $^{3,5}$ | \\ Celine T. Aquino ${ }^{6}$ । Linda S. Adair ${ }^{7}$ | Thomas W. McDade ${ }^{1,2}$ (])
}

${ }^{1}$ Department of Anthropology, Northwestern

University, Evanston, Illinois

${ }^{2}$ Institute for Policy Research, Northwestern

University, Evanston, Illinois

${ }^{3}$ USC-Office of Population Studies Foundation, Inc., University of San Carlos, Cebu City, Philippines

${ }^{4}$ Department of Nutrition and Dietetics, University of San Carlos, Cebu City, Philippines

${ }^{5}$ Department of Anthropology, Sociology and History, University of San Carlos, Cebu City, Philippines

${ }^{6}$ Department of Medicine, Cebu Institute of Medicine, Cebu City, Philippines

${ }^{7}$ Carolina Population Center and Department of Nutrition, University of North Carolina, Chapel Hill, North Carolina

Correspondence

Christopher W. Kuzawa, Department of Anthropology, Northwestern University, 1810

Hinman Ave, Evanston, IL 60208.

Email: kuzawa@northwestern.edu

Funding information

National Institutes of Health, Grant/Award

Number: R01AG039443

\begin{abstract}
Objective: Cardiovascular disease (CVD) is rising in low and middle-income countries, but studies of CVD epidemiology in such settings often focus on risk factors rather than measures of disease progression. Here we use the ankle brachial index (ABI) to assess the prevalence of peripheral artery disease (PAD) among older women living in Metropolitan Cebu, Philippines, and relationships between $\mathrm{ABI}$ and CVD risk factors and body composition.

Methods: ABI was measured using the Doppler technique in 538 female participants in the 2015 Cebu Longitudinal Health and Nutrition Survey (mean age 58 years, range 47-78 years). ABI was related to a panel of CVD risk factors measured in 2005 and 2012, and to 2012 body composition measures.

Results: The prevalence of PAD (1.8\%) was among the lowest reported in any comparably-aged sample, and only $9.9 \%$ of participants had an ABI indicating borderline PAD risk. Smoking $(P<0.011)$ and use of CVD medications $(P<0.0001)$ predicted lower ABI (indicating higher PAD risk), which was also lower in relation to 2012 systolic blood pressure $(P<0.054)$. ABI was unrelated to other CVD risk factors. An apparent protective relationship between body mass index (BMI) and $\mathrm{ABI}$, noted in previous studies, was found to be confounded by protective relationships between $\mathrm{ABI}$ and fat free mass, height, and grip strength (all $P<0.05$ ).

Conclusions: The prevalence of PAD is low in Cebu Longitudinal Health and Nutrition Survey participants, and ABI was related to few CVD risk factors. Past reports of lower PAD risk in relation to BMI may reflect confounding by lean mass, which has protective relationships with $\mathrm{ABI}$.
\end{abstract}

\section{1 | INTRODUCTION}

Cardiovascular disease (CVD) is a leading cause of morbidity and mortality worldwide (Lozano et al., 2012; Roth et al., 2015), and CVD-related deaths are projected to rise from 16.7 million in 2002 to 23.3 million in 2030 (Mathers \& Loncar, 2006). This increase is particularly dramatic in lowand middle-income countries in Southeast Asia that are undergoing nutrition and lifestyle transitions, with rising rates of overweight and obesity contributing to the increasing burden of chronic metabolic disease (Popkin, 2001).
Despite this growing CVD burden, most studies examining the etiology of metabolic disease in this context focus on intermediary risk factors such as lipids, glucose, and blood pressure rather than measuring disease progression itself. While direct measures such as coronary artery calcification tests are effective in capturing disease progression, their cost and reliance on imaging equipment limits their utility in field-based studies (Wexler et al., 1996). In contrast, ankle brachial index (ABI) is a noninvasive and field-friendly measure that captures the progression of atherosclerosis in the lower limbs in both symptomatic and asymptomatic 
individuals (Aboyans et al., 2012). Arterial stiffening in the lower limbs-also referred to as peripheral artery disease (PAD) - is a leading cause of cardiovascular mortality with a growing global prevalence (Caro, Migliaccio-Walle, Ishak, \& Proskorovsky, 2005; Criqui et al., 1992; Fowkes et al., 2016). ABI is thus a potentially useful tool for investigating the impact of rapid nutritional and lifestyle change on the development of atherosclerosis in community-based settings around the world.

The Philippines is a lower-middle-income country in Southeast Asia where cardiovascular risk factors, including overweight and obesity, are changing in the context of a transitioning economy marked by increasing urbanization (Adair, 2004; World Bank, 2018). In a recent Philippines national survey of more than 19000 adults aged 20 and above, only $0.1 \%$ reported a history of PAD (FNRI-DOST, 2015b). However, $83.4 \%$ of the sample had never heard of the disease and costs associated with diagnosing the condition may often be prohibitive. Here we use Doppler ultrasound measured ABI to characterize PAD progression among women (48-79 years old) participating in a study of aging in Metropolitan Cebu, Philippines. Our past work tracked the emergence of CVD risk factors among this cohort as they aged, including evidence for secular trends, and explored the role of changes in diet and weight gain (Adair, Kuzawa, McDade, Carba, \& Borja, 2018). Our goals in the present analyses are to use ABI, measured in 2015, to describe the prevalence of PAD in this population, and to report links between the $\mathrm{ABI}$ and a panel of CVD risk factors and body composition measures obtained in 2012 and 2005.

\section{I METHODS}

The Cebu Longitudinal Health and Nutrition Survey (CLHNS) began in 1983 with recruitment of 3327 pregnant women from randomly selected urban and rural neighborhoods in Metropolitan Cebu, Philippines (Adair et al., 2011). The analyses presented in this article include data collected among these women during follow-up surveys in 2005 and 2012, which are related to ABI measured in 2015. Socioeconomic, demographic, health behavior, and anthropometric information were collected during in-home interviews. Complete ABI, anthropometric, and sociodemographic data were available on a subsample of 538 participants. When compared to women visited in 2012 but excluded from the ABI analysis, the ABI subsample was slightly heavier $(57.2 \mathrm{~kg}$ vs $55.9, P<0.031)$, had a higher BMI $\left(25.3 \mathrm{vs} 24.7 \mathrm{~kg} / \mathrm{m}^{2}\right.$, $P<0.011$ ) and waist circumference (WC; 83.0 vs $81.6 \mathrm{~cm}$, $P<0.03$ ), and had borderline lower HbAlc (5.8 vs 5.9\%, $P<0.07)$, but had similar household assets, heights, systolic and diastolic blood pressures, and total and HDL cholesterol levels (all $P>0.1$ ). We also assessed whether the ABI analysis subsample had CVD risk factors measured in 2005 (10 years prior to ABI measurement) that differed from women excluded from the study. In 2005, the women included in the ABI analyses had modestly lower systolic blood pressure (118.4 vs $120.1 \mathrm{~mm} \mathrm{Hg}, P<0.093$ ), diastolic blood pressure (78.7 vs $80.0 \mathrm{~mm} \mathrm{Hg}, P<0.048$ ), and fasting glucose (95.4 vs $101.1 \mathrm{mg} / \mathrm{dL}, P<0.002)$, but had similar total cholesterol, HDL cholesterol, and C-reactive protein (CRP; all $P>0.13$ ). All research was conducted under conditions of written informed consent with Institutional Review Board oversight by the University of North Carolina, Chapel Hill and the University of San Carlos.

\section{1 | Behavioral and socioeconomic data}

Socioeconomic status was indexed by a household assets scale reflecting whether the family had electricity, owned homes made of strong material, owned an air conditioner, refrigerator, TV, vehicle and other appliances. Women were also asked about their highest completed level of education. Women were defined as smokers if they reported smoking at least 1 cigarette daily.

\section{2 | Medication use}

During the 2012 interview, women were asked about each medication that they were currently taking, and were classified as taking a CVD-related drug if they were taking any drug included in MIMS classification for treatment of CVDs (MIMS 200-299): cardiac drugs, anti-anginal drugs, antihypertensives (eg, diuretics, beta blockers), peripheral vasodilators and cerebral activators, vasoconstrictors, dyslipidemic agents, hemostatics, anticoagulants, antiplatelets and fibrinolytics (thrombolytics), phlebitis and varicose preparations, hemorrheologicals, hematopoietic agents, other cardiovascular drugs, along with insulin preparations (MIMS 1101), and antidiabetic agents (MIMS 1102).

\subsection{Anthropometry, body composition, and grip strength}

In 2005 and 2012, weight, height, and WC were measured using standard techniques (Lohman, Roche, \& Martorell, 1991). The body mass index (BMI) was calculated as $\mathrm{kg} / \mathrm{m}^{2}$, and individuals were classified as overweight if BMI $>25$ $\mathrm{kg} / \mathrm{m}^{2}$ or obese if $\mathrm{BMI}>30 \mathrm{~kg} / \mathrm{m}^{2}$. In 2012, we also obtained estimates of percentage body fat using a Tanita bioelectrical impedance scale (algorithms adjusted for Asian populations), which was used (along with weight) to calculate fat mass and fat-free mass. Grip strength was measured with a Smedley-type dynamometer in triplicate in each hand. Left and right grip strengths were defined as the highest reading for that hand. In analyses, we included grip strength of the dominant hand, defined as the hand with the stronger reading.

\section{4 | Ankle-brachial index}

The ABI protocol was administered as part of the 2015 CLHNS round. ABI recruitment was limited to women who 
had participated in the 2005 (and who provided biomarker data), 2012, and 2015 surveys. Because the protocol required ABI to be measured in health centers, we only recruited mothers who had already attended clinic sessions as part of the 2012 CLHNS flu vaccination protocol. ABI measurements and calculations followed the standard procedure detailed by the American Heart Association (Aboyans et al., 2012). Trained technicians conducted the ABI measurements at a health center near participants' homes during the 2015 followup. They measured systolic blood pressure twice at each ankle and at the brachial artery in each arm using the Doppler technique (Hadeco ES100V3 Bidop Arterial and Venous Doppler with Smart V-Link software). The ABI was calculated as the ratio of systolic blood pressure measured at the ankle to that measured at the brachial artery in the upper arm, averaging the two measures for each limb and using the arm with the highest blood pressure measurement. ABI values $\leq 0.90$ indicate that an individual has PAD and is at high risk for future cardiac events such as myocardial infarction. ABI values that are $>0.90$ but $\leq 1.0$ indicate increased risk for PAD and moderate risk for future cardiac events (Aboyans et al., 2012). High ABI values $(>1.4)$ are interpreted as evidence for arterial stiffening due to calcification (Resnick, 2004).

\section{5 | Blood pressure}

Systolic and diastolic blood pressures were measured in triplicate, after 10 minutes of seated rest, by trained research staff using appropriate cuff sizes, and using OMRON HEM7211 blood pressure monitors. Following International Diabetes Foundation guidelines (Alberti, Zimmet, Shaw, \& Grundy, 2006), individuals were classified as having elevated blood pressure (EBP) if they met one or more of the following criteria: systolic blood pressure $\geq 130 \mathrm{~mm} \mathrm{Hg}$, diastolic blood pressure $\geq 85 \mathrm{~mm} \mathrm{Hg}$, or taking antihypertensive medications.

\section{6 | C-reactive protein (CRP)}

In 2005, venipuncture blood samples were collected using EDTA-coated vacutainer tubes in the participants' homes in the morning after the overnight fast. Blood samples were kept in coolers on ice packs for no more than 2 hours and were then centrifuged to separate plasma prior to freezing at $-35 \mathrm{C}$. Samples were express-shipped in a single batch to Northwestern University on dry ice and stored frozen at $-80 \mathrm{C}$ until analysis at the clinical chemistry facility at Northwestern Memorial Hospital in Evanston. CRP concentrations were determined using a high sensitivity immunoturbidimetric method (Synchron LX20, lower detection limit: $0.1 \mathrm{mg} / \mathrm{L}$ ). In 2012, finger stick capillary blood samples were collected on filter paper for analysis of CRP. The participant's finger was cleaned with alcohol, and a sterile, disposable microlancet was used to extract drops of blood that were applied to filter paper (McDade, Williams, \& Snodgrass, 2007). After collection, DBS cards were dried for at least 4 hours before being stored in sealed bags at $-20 \mathrm{C}$ until express shipment to Northwestern University, where they were stored and analyzed (see McDade, Borja, Kuzawa, Perez, \& Adair, 2015 for details). DBS samples were analyzed for CRP in the Laboratory for Human Biology Research using a modified high sensitivity enzyme immunoassay protocol previously developed for use with DBS (McDade, Burhop, \& Dohnal, 2004). CRP was analyzed as a continuous variable and was also used to classify individuals as having high CRP, defined as a CRP $\geq 3$ (but less than 10, which may be interpreted as an active infection; Pearson et al., 2003).

\section{7 | Measures of glucose control}

In 2005, fasting glucose was measured at the time of venipuncture with one drop of blood using a portable glucose meter (One Touch Ultra Blood Glucose Monitoring System, Johnson and Johnson) and with values corrected to align with capillary samples (for further protocol details see Norris et al., 2012), and with diabetes defined as a value $\geq 7 \mathrm{mmol} / \mathrm{L}$. In 2012, HbA1c was measured in capillary finger prick blood samples using the NycoCard Reader II, with diabetes defined as an A1c value of at least 6.5\% (International Expert Committee, 2009).

\section{8 | Lipid profiles}

In 2005, lipid profiles were measured in plasma collected after an overnight fast, and were shipped frozen on dry ice to the Emory Lipid Research Laboratory for analysis. Total lipids were determined by enzymatic methods using reagents from Beckman Diagnostics (Palo Alto, California) on a CX5 chemistry analyzer. HDL cholesterol was determined using the homogenous assay for direct HDL cholesterol (Genzyme Corporation, Exton, Pennsylvania). In 2012, nonfasting lipid profiles (total cholesterol, HDL cholesterol) were measured in capillary finger prick blood samples using the CardioChek point-of-care system, which has been validated against venous blood measures (Ferreira et al., 2015).

\section{9 | Statistical analyses}

We first calculated descriptive statistics for $\mathrm{ABI}$ to compare the prevalence of PAD among women in the present sample with findings from previous studies. We then ran a series of predictive regression models aimed at clarifying the links between $\mathrm{ABI}$ and body composition measures. The first model adjusted only for age. Because ABI was significantly lower among smokers $(1.046+/ 0.114$ vs $1.078 \pm 0.073$; $P<0.011)$ and among individuals taking medications for CVDs $(1.049+/ 0.113$ vs $1.081 \pm 0.067 ; P<0.0001)$, we ran a second model adjusted for both. We also included an assets scale in this model, which was not a significant predictor of ABI $(P<0.21)$ but did modify model coefficients. To assess the possible role of lean tissue and strength on 
TABLE 1 Characteristics of sample in 2005 and $2012(\mathrm{~N}=538)$

\begin{tabular}{|c|c|c|c|c|}
\hline \multirow[b]{2}{*}{ Variable } & \multicolumn{2}{|l|}{2005} & \multicolumn{2}{|l|}{2012} \\
\hline & Mean (SD) & Range & Mean (SD) & Range \\
\hline Age (years) & $47.9(5.9)$ & $(37.2,69.3)$ & $55.2(5.8)$ & $(44.5,75.9)$ \\
\hline Weight $(\mathrm{kg})$ & $56.0(10.5)$ & $(31,92.3)$ & $57.2(11.4)$ & $(31.3,103.1)$ \\
\hline BMI $\left(\mathrm{kg} / \mathrm{m}^{2}\right)$ & $24.7(4.2)$ & $(14.5,38.3)$ & $25.3(4.6)$ & $(14.2,42.9)$ \\
\hline Waist circum. (cm) & $82.3(10.4)$ & $(59,118.4)$ & $83.0(11.4)$ & $(51.4,123.3)$ \\
\hline Obese $(\%)$ & $9.9 \%$ & & $13.2 \%$ & \\
\hline Smoker (\%) & & & $7.4 \%$ & \\
\hline Grades completed & $6.9(3.2)$ & $(0,17)$ & & \\
\hline Taking CVD drug (\%) & & & $16.2 \%$ & \\
\hline Systolic blood pressure $(\mathrm{mm} \mathrm{Hg})$ & $118.4(18.1)$ & $(80,190)$ & $127.7(20.1)$ & $(82.3,194.7)$ \\
\hline Total cholesterol (mg/dL) & $187.7(37.9)^{\mathrm{c}}$ & $(89,393)$ & $199.7(37.7)$ & $(108,379)$ \\
\hline HDL cholesterol (mg/dL) & $40.3(10.0)^{\mathrm{c}}$ & $(15.7,80.7)$ & $43.6(12.9)$ & $(17,94)$ \\
\hline Hbalc $(\%)$ & & & $5.8(1.2)$ & $(4.4,13.4)$ \\
\hline Glucose $(\mathrm{mmol} / \mathrm{L})$ & $5.30(1.64)$ & $(3.36,20.03)$ & & \\
\hline Diabetic $(\%)^{\mathrm{d}}$ & $5.4 \%$ & & $10.0 \%$ & \\
\hline
\end{tabular}

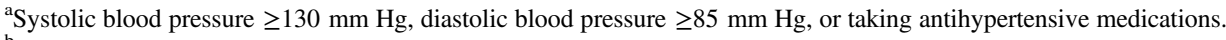

${ }^{\mathrm{b}} \mathrm{n}=535$.

${ }^{c} \mathrm{n}=536$.

${ }^{\mathrm{d}}$ Fasting glucose $\geq 7 \mathrm{mmol} / \mathrm{L}$ (2005) and Hbalc $\geq 6.5 \%$ (2012).

ABI, the third, fourth, and fifth models built on model 2 and alternatively included height, fat free mass and grip strength. Finally, we ran regression models (model 1 adjusting only for age, model 2 adjusting for age, smoking status, use of CVD-related drugs and household assets) linking a series of CVD risk factors measured in 2005 and 2012, and risk factor status (overweight or obese, diabetic, EBP, and elevated CRP), with ABI measured in 2015. Models linking CRP to ABI were run after excluding individuals whose CRP levels were above 10, suggesting an active infection. All analyses were performed using Stata version 13.1 for IBM (StataCorp, College Station, Texas).

\section{3 | RESULTS}

This study describes ABI measured in 2015 in relation to CVD risk factors and other covariates measured in 2012 and 2005. The mean ABI of the sample (age $58.2 \pm 5.8$ years, range $47.5,78.7$ years) was $1.08 \pm 0.08$ (range $0.54,1.38$ ) Characteristics of the sample during the 2005 and 2012 surveys are presented in Table 1 . Ten participants (1.86\% of the sample) had ABI values $\leq 0.90$ indicating current $\mathrm{PAD}$, and no participants had elevated ABI $(\mathrm{ABI}>1.4)$ consistent with arterial stiffening (Figure 1). Fifty-three participants (9.9\% of the sample) were classified as having borderline risk for PAD with $\mathrm{ABI}$ values $>0.90$ and $\leq 1.0$, and the remaining 475 participants $(88.3 \%$ of the sample) were at low risk for PAD with $\mathrm{ABI}$ values $>1.0$. There was no indication that $\mathrm{ABI}$ or PAD risks were increased at older ages (Figure 2). Among the 19 individuals who were 70 or older, the mean ABI was 1.039 (SD 0.094) and two (10.5\%) had an ABI consistent with PAD. Considered on their own, smoking status $(-0.032 \pm 0.013 ; P<0.011)$ and cardiovascular medication use $(-0.032 \pm 0.009 ; P<0.0001)$ were significant predictors of lower ABI, but ABI was not significantly related to the woman's age (in years) at ABI measurement $(-0.001 \pm 0.0006 ; P<0.121)$ or her household assets score (scale range $1-11 ; 0.003 \pm 0.002 ; P<0.209$ ).

Results of linear regression models predicting $\mathrm{ABI}$ as a continuous variable are shown in Tables $2-4$. After adjustment for age, household assets, smoking status and CVD-related drug use, BMI, WC, and fat mass measured in 2012 were all positively related to $\mathrm{ABI}$ (each run separately), consistent with a weak protective relationship. Although none of these relationships were significant, the $P$-value for all was $<0.2$ and fat mass was a borderline significant $(P<0.059)$ positive predictor of ABI. We next added, sequentially, height, fat free mass, and grip strength to the adjusted models to evaluate the potential role of fat free mass or strength as confounders. Height, fat free mass, and grip strength were all significant or borderline significant positive predictors of $\mathrm{ABI}$ in these models, and adjusting for these relationships substantially attenuated the relationships between $\mathrm{ABI}$ and the BMI, WC, and fat mass.

We next ran models assessing relationships between $\mathrm{ABI}$ and a panel of cardiovascular risk factors measured in 2012 . 


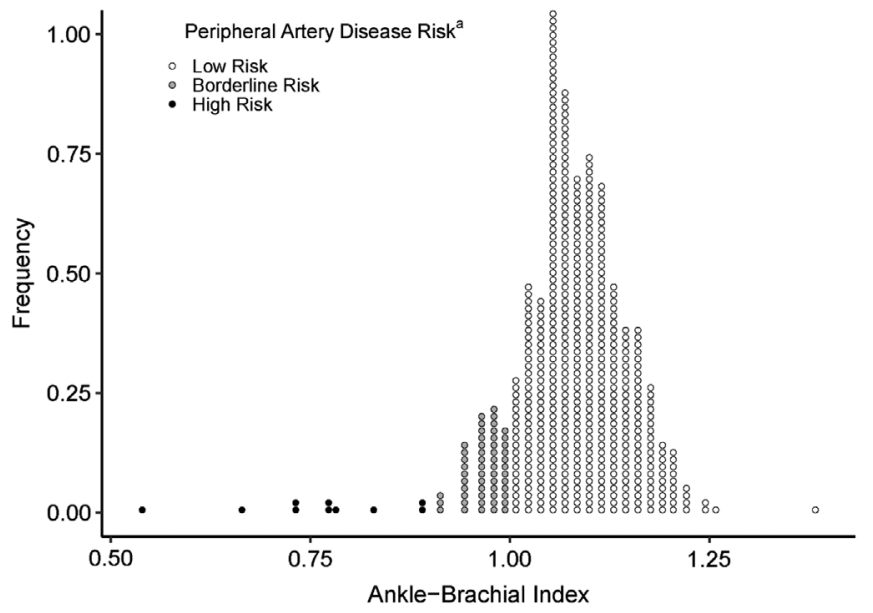

FIGURE 1 Distribution of ankle-brachial index (ABI) values for women in the Cebu Longitudinal Health and Nutrition Survey. ${ }^{\text {a Risk categories }}$ (Aboyans et al., 2012): "Low Risk" = ABI >1.0, "Borderline Risk" $=0.90<$ $\mathrm{ABI} \leq 1.0$, "High Risk" $=\mathrm{ABI} \leq 0.90$. High risk values indicate diagnosable peripheral artery disease and a high likelihood of future cardiac events and cardiovascular-related mortality

Model 1 adjusted only for age while model 2 adjusted for age, household assets, smoking status, and CVD-related drug use. Models explained relatively little variance in ABI: all model 1 adjusted $R^{2}$ were less than 0.015 , whereas model 2 adjusted $R^{2}$ varied from 0.032 to 0.036 (not shown). Of the factors considered, only systolic blood pressure and the closely-related measure of EBP were significant predictors of lower ABI (high PAD risk) in minimally-adjusted models. After additional adjustments in model 2, systolic blood pressure dropped to a borderline significant relationship $(P<0.053)$ and EBP was no longer significant $(P>0.2)$. No other CVD risk factors predicted $\mathrm{ABI}$ in minimally or

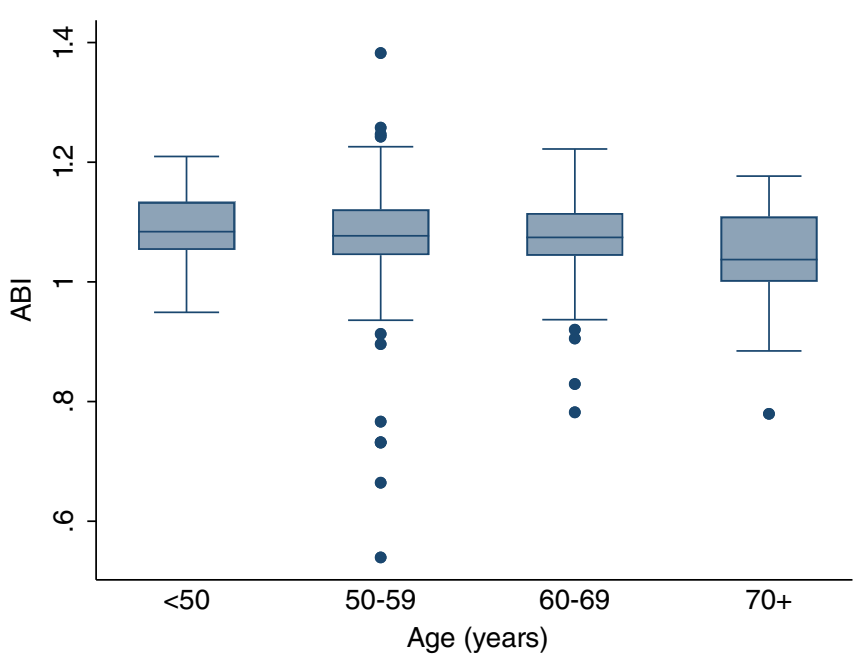

FIGURE 2 Distribution of ABI by age category among Cebu women. Sample sizes for the four groups (in order of ascending age) were 22, 332, 165 , and 22

fully adjusted models. Table 4 reports results of comparable models linking ABI to CVD risk factors measured in 2005; only systolic blood pressure was a significant predictor in unadjusted models, and no CVD risk factor predicted $\mathrm{ABI}$ in adjusted models.

\section{4 | DISCUSSION}

In this aging cohort of women from Cebu, Philippines, we find a relatively high average $\mathrm{ABI}$, and corresponding low prevalence of PAD, compared with most prior studies of similarlyaged women. Risk for PAD was increased in relation to systolic blood pressure, and among smokers and individuals

TABLE 2 Standardized regression coefficients from regression models linking 2012 body composition to $2015 \mathrm{ABI}^{\mathrm{a}}$

\begin{tabular}{|c|c|c|c|c|c|}
\hline & Base model $^{\mathbf{b}}$ & Adjusted model $^{\mathbf{c}}$ & +Height & + FFM & +Grip strength \\
\hline BMI $\left(\mathrm{kg} / \mathrm{m}^{2}\right)$ & $0.044(0.044)$ & $0.060(0.045)$ & $0.054(0.045)$ & $-0.032(0.062)$ & $0.034(0.047)$ \\
\hline Height $(\mathrm{cm})$ & & & $0.092(0.043)^{*}$ & & \\
\hline Grip strength (kg) & & & & & $0.102(0.046)^{*}$ \\
\hline Model adj. $R^{2}$ & 0.003 & 0.035 & 0.041 & 0.041 & 0.042 \\
\hline Waist circumference & $0.065(0.043)$ & $0.085(0.045)$ & $0.068(0.046)$ & $0.008(0.065)$ & $0.064(0.046)$ \\
\hline Fat free mass $(\mathrm{kg})$ & & & & $0.106(0.066)$ & \\
\hline Grip strength (kg) & & & & & $0.096(0.045)^{*}$ \\
\hline Model adj. $R^{2}$ & 0.0049 & 0.038 & 0.043 & 0.041 & 0.044 \\
\hline Fat mass $(\mathrm{kg})$ & $0.050(0.044)$ & $0.066(0.045)$ & $0.046(0.046)$ & $-0.030(0.065)$ & $0.038(0.047)$ \\
\hline Height (cm) & & & $0.085(0.044)^{\wedge}$ & & \\
\hline
\end{tabular}

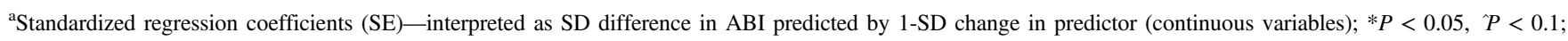
FFM, fat free mass.

${ }^{\mathrm{b}}$ Adjusted for maternal age (coefficient not shown).

${ }^{\mathrm{c}}$ Adjusted for maternal age, highest education level, smoking status and use of cardiovascular drugs. 
TABLE 3 Standardized regression coefficients from models relating ABI (2015) to individual cardiovascular disease risk factors (2012) ${ }^{\mathrm{a}}$

\begin{tabular}{|c|c|c|}
\hline & Base models ${ }^{\mathbf{b}}$ & Adjusted models ${ }^{c}$ \\
\hline Systolic blood pressure & $-0.120(0.043)^{* *}$ & $-0.085(0.044)^{\wedge}$ \\
\hline Diastolic blood pressure & $-0.041(0.043)$ & $-0.012(0.043)$ \\
\hline Elevated blood pressure $^{\mathrm{d}}$ & $-0.203(0.087)^{*}$ & $-0.11(0.093)$ \\
\hline $\mathrm{CRP}^{\mathrm{e}}$ & $-0.102(0.081)$ & $-0.113(0.081)$ \\
\hline High $\mathrm{CRP}^{\mathrm{e}, \mathrm{f}}$ & $-0.085(0.112)$ & $-0.111(0.112)$ \\
\hline Total cholesterol & $-0.035(0.043)$ & $-0.034(0.043)$ \\
\hline HDL cholesterol & $-0.026(0.043)$ & $-0.036(0.043)$ \\
\hline $\mathrm{HbA1c}$ & $-0.037(0.043)$ & $-0.019(0.043)$ \\
\hline Diabetic ${ }^{\mathrm{g}}$ & $-0.127(0.143)$ & $-0.027(0.146)$ \\
\hline
\end{tabular}

${ }^{\mathrm{a}}$ Standardized regression coefficients (SE)—interpreted as SD difference in ABI predicted by 1-SD change in predictor (continuous variables) and SD difference in ABI predicted by risk category status (categorical variables) $* * P<0.01$, $* P<0.05, \stackrel{P}{P}<0.1$

${ }^{\mathrm{b}}$ Adjusted for maternal age (coefficient not shown).

${ }^{\mathrm{c}}$ Adjusted for maternal age, highest education level, smoking status and use of cardiovascular drugs (coefficients not shown).

${ }^{\mathrm{d}}$ Systolic blood pressure $\geq 130 \mathrm{~mm} \mathrm{Hg}$, diastolic blood pressure $\geq 85 \mathrm{~mm} \mathrm{Hg}$, or taking antihypertensive medications.

${ }^{\mathrm{e}}$ Excludes 7 individuals with CRP $\geq 10 \mathrm{mg} / \mathrm{dL}$.

${ }^{\mathrm{f}} \mathrm{CRP} \geq 3$.

${ }^{\mathrm{g}} \mathrm{Hbalc} \geq 6.5 \%$

taking medications for CVDs, but was unrelated to most biomarkers of CVD risk measured at two time points in the years preceding $\mathrm{ABI}$ measurement. Women who were taller or who had a greater fat free mass had an ABI consistent with lower $\mathrm{PAD}$ risk, but the $\mathrm{ABI}$ was unrelated to measures of adiposity. These findings point to an unusually low burden of PAD in the context of a population undergoing the nutrition transition and rising CVD (FNRI-DOST, 2015a; Pedro, Barba, \& Leon, 2007).

Only 10 participants in the present study (1.9\%) had ABI values indicating current PAD. The only study of a

TABLE 4 Standardized regression coefficients from models relating ABI (2015) to individual cardiovascular disease risk factors (2005) ${ }^{\mathrm{a}}$

\begin{tabular}{|c|c|c|}
\hline & Base models $^{\mathbf{b}}$ & Adjusted models $^{c}$ \\
\hline Systolic blood pressure & $-0.104(0.044)^{*}$ & $-0.054(0.047)$ \\
\hline Diastolic blood pressure & $-0.044(0.044)$ & $-0.004(0.045)$ \\
\hline Elevated blood pressure $^{\mathrm{d}}$ & $-0.130(0.089)$ & $-0.021(0.093)$ \\
\hline $\mathrm{CRP}^{\mathrm{e}}$ & $-0.071(0.131)$ & $-0.041(0.129)$ \\
\hline High $C_{R P} P^{e, f}$ & $0.004(0.110)$ & $0.016(0.108)$ \\
\hline Total cholesterol & $-0.051(0.044)$ & $-0.053(0.043)$ \\
\hline HDL cholesterol & $0.040(0.043)$ & $0.034(0.043)$ \\
\hline Glucose & $-0.041(0.043)$ & $-0.027(0.043)$ \\
\hline Diabetic $^{g}$ & $-0.210(0.191)$ & $-0.118(0.191)$ \\
\hline
\end{tabular}

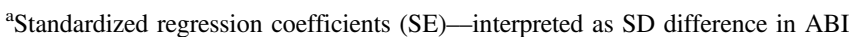
predicted by 1-SD change in predictor (continuous variables) and SD difference in ABI predicted by risk category status (categorical variables); beta coefficient (SE) $* * P<0.01, * P<0.05, \stackrel{\wedge}{\wedge}<0.1$

${ }^{\mathrm{b}}$ Adjusted for maternal age (coefficient not shown).

${ }^{\mathrm{c}}$ Adjusted for maternal age, highest education level, smoking status, and use of cardiovascular drugs (coefficients not shown).

${ }^{\mathrm{d} S y s t o l i c ~ b l o o d ~ p r e s s u r e ~} \geq 130 \mathrm{~mm} \mathrm{Hg}$, diastolic blood pressure $\geq 85 \mathrm{~mm} \mathrm{Hg}$, or taking antihypertensive medications.

${ }^{\mathrm{e}}$ Excludes 15 individuals with $\mathrm{CRP} \geq 10 \mathrm{mg} / \mathrm{dL}$.

${ }^{\mathrm{f}} \mathrm{CRP} \geq 3$.

${ }^{\mathrm{g}}$ Fasting glucose $\geq 7 \mathrm{mmol} / \mathrm{L}$. similarly-aged cohort that we are aware of that reported a lower prevalence was a study of forager-horticulturalists living in lowland Bolivia, in which no participants had an ABI consistent with PAD (Gurven et al., 2009). The lowest prevalence according to $\mathrm{ABI}$ values recorded in a meta-analysis conducted by Fowkes, Murray, and Butcher (2008) was 2.8\% reported in 20 to 74 year old women $(\mathrm{N}=838)$ participating in the San Luis Valley Diabetes Study in Colorado. In a recent review of studies measuring ABI among countries in SubSaharan Africa, Johnston et al. (2016) found a range of PAD prevalence ranging from $3.1 \%$ in a rural horticulturalist population in Ghana to $52.2 \%$ for a sample of older adults with diabetes in Nigeria. Across Asia, studies have reported ABI levels consistent with PAD in $11 \%$ of women in Hong Kong (age $72.6 \pm 5.4$ years), $17.7 \%$ in Beijing (male and female mean age $68.5 \pm 5.4$ years), and $8.6 \%$ in Thailand (age range 52-73 years; He et al., 2006; Sritara et al., 2007; Woo et al., 2006).

We found that ABI was not related to age in the CLHNS sample, and there was also minimal evidence for links between other CVD risk factors and ABI. Of the panel of CVD risk factors that we considered, only systolic blood pressure (and the closely related measure of EBP), measured 3 years prior to $\mathrm{ABI}$ measurement, was a borderline significant predictor of lower ABI. In contrast to findings in most other studies, we found that lipid profiles, measures of glucose control and diabetes status, and inflammation were not significant predictors of $\mathrm{ABI}$, with or without additional controls for socioeconomic and other factors. This pattern of findings is most consistent with the study of Tsimane foragerhorticulturalists from lowland Bolivia noted above. In this sample PAD was completely absent in all age groups, and the $\mathrm{ABI}$ was found to be unrelated to the BMI or obesity, lipid profiles, systolic blood pressure and several measures of inflammation (Gurven et al., 2009). Of the CVD risk factors considered, only diastolic blood pressure was a significant, inverse predictor of ABI. The authors of the Tsimane study speculate that physical activity, genetic variants specific to Amerindian populations, or differences in infectious disease ecology, such as chronic exposure to helminths, could protect the population against PAD. Our prior work with the CLHNS has shown that early life infectious or microbial exposures predict lower CRP in the adult offspring of the mothers included in this study, which could point to differences in inflammatory regulation among CLHNS participants (McDade, Rutherford, Adair, \& Kuzawa, 2010). Although speculative, a higher ambient pathogen exposure in the Tsimane and Cebu samples, compared to other samples that have been the focus of work on PAD, may limit the prevalence of PAD.

Consistent with prior studies (reviewed in Criqui \& Aboyans, 2015), we found that adiposity measures such as the BMI, WC, and fat mass were not significant predictors of the ABI in fully adjusted models. It is notable that, when assessed on their own, all three measures were weakly 
positively related to $A B I$, suggesting weak protective relationships with $\mathrm{ABI}$ and PAD risk, with WC closest to significance $(P<0.136)$. Despite robust evidence for a link between adiposity or obesity and CVD (Hajer, Haeften, W, \& Visseren, 2008), a majority of past studies of PAD have either failed to find relationships between BMI and $\mathrm{ABI}$, or found evidence that higher BMI is associated with lower PAD risk (Criqui \& Aboyans, 2015). For instance, working with US NHANES data, Selvin (2004) found that obese individuals (BMI $>30 \mathrm{~kg} / \mathrm{m}^{2}$ ) were roughly half as likely to have PAD compared to individuals who were normal weight or underweight (BMI $<25 \mathrm{~kg} / \mathrm{m}^{2}$ ). Similarly, among 5084 participants (age $>65$ years) in the US-based Cardiovascular Health Study, the BMI was again significantly related to lower PAD risk (Newman et al., 1993). As in Cebu, a study in Thailand found BMI to be a significant positive predictor of $\mathrm{ABI}$, consistent with lower PAD risk (Sritara et al., 2007).

Explanations for the finding of an apparent protective relationship between measures like $\mathrm{BMI}$ or $\mathrm{WC}$ and $\mathrm{PAD}$ risk in past studies have focused on the potential confounding role of smoking and/or poor health, both of which may be associated with atherosclerosis progression while also leading to weight loss (Criqui \& Aboyans, 2015). In a follow-up analysis of the US Cardiovascular Health Study described above (Newman et al., 1993), Ix et al. (2011) limited analyses to healthy nonsmokers and found a positive relationship between BMI and PAD risk in this subsample. Although the prevalence of smoking is relatively low in our sample, adjusting for smoking in models did modestly attenuate relationships between ABI and measures of adiposity, pointing to the potential for similar confounding of associations in the CLHNS sample.

Availability of bioelectrical impedance data from the 2012 survey allowed us to calculate fat and fat free mass, which provided additional insights into the relationship between body composition and PAD risk. We found that both height and lean mass were significant positive predictors of $\mathrm{ABI}$, and that the nonsignificant positive relationships with adiposity measures were all weakened substantially by their inclusion in models. In aging samples, reduced muscle mass and strength have previously been shown to be related to pro-inflammatory cytokines (Visser et al., 2002), reduced insulin sensitivity (Srikanthan \& Karlamangla, 2011), and arterial stiffness (Ochi et al., 2010), hinting at possible mechanisms that could contribute to these relationships. One potential interpretation of our body composition findings is that muscle mass has a protective relationship with $\mathrm{ABI}$ and that the tendency for lean mass and measures of adiposity to be positively related confounds associations with these other measures. In follow-up analyses, we found that grip strength was even stronger than lean mass as a positive predictor of ABI, consistent with possible involvement of muscle and strength in these relationships. These findings could help explain the common reports, noted above, of positive relationships between measures of adiposity like BMI and the $\mathrm{ABI}$ in other populations. If this finding is replicated and shown to be causal, it also points to the potential importance of promoting muscle mass, such as through resistance training (Hunter, McCarthy, \& Bamman, 2004), as a means of protecting against age-related development of PAD in older women.

Several limitations of this study warrant mention. Most notably, because this was a follow-up of a birth cohort study that enrolled pregnant women in 1983, we focus solely on women, and our findings should not be extrapolated to men. Because our sample had a mean age of 55 years, prevalence of PAD could rise further as the sample ages. Finally, this study involved a single cross-sectional ABI measurement and, due to the logistical constraints of clinic visits, involved a convenience sub-sample of the full CLHNS sample. Because the women included in the ABI sub-sample had lower blood pressure and glucose values 10 years prior to ABI measurement, when compared to women excluded from the ABI sub-study (see Section 2), it is possible that the prevalence of PAD that we document slightly underestimates levels that would be seen in a representative sample from Cebu. These study design issues limit our ability to explore causal relationships longitudinally, and also limit the sample's representativeness of the Cebu population from which they were drawn.

In sum, we find evidence for a very low prevalence of PAD in an aging cohort of women living in Metropolitan Cebu, Philippines. These findings are consistent with the low reported prevalence of the condition in a recent national survey in the Philippines (FNRI-DOST, 2015b). Risk for PAD was increased among smokers, among women taking medications for CVD, and in relation to systolic blood pressure. Fat free mass and grip strength were found to have significant, protective relationships with ABI. Our findings are most similar to a study in lowland Bolivia in which PAD was absent, did not increase with age and was unrelated to body composition or most CVD risk factors. We speculate that shared features of the ecologies experienced by these populations, perhaps including early infectious disease exposure, could help explain these findings.

\section{ACKNOWLEDGMENTS}

The authors thank the many researchers at the USC-Office of Population Studies, University of San Carlos, Cebu, Philippines, for their role in study design and data collection, and the Filipino participants, who generously provided their time for this study. Fieldwork and sample collection were supported by NIH R01AG039443 (2012 and 2015 data), the Interdisciplinary Obesity Center (RR20649) and the Center for Environmental Health and Susceptibility (ES10126; project 7-2004-E)(2005 data). 


\section{AUTHOR CONTRIBUTIONS}

CWK and TMB conducted the analysis and wrote the manuscript; LSA, CWK, TWM, JBB, NRL, and CTA conceived of the study and facilitated data collection; all authors commented on and approved the manuscript.

\section{ORCID}

Christopher W. Kuzawa (1) https://orcid.org/0000-00020649-8677

\section{Thomas W. McDade (10) https://orcid.org/0000-0001-5829- $648 \mathrm{X}$}

\section{REFERENCES}

Aboyans, V., Criqui, M. H., Abraham, P., Allison, M. A., Creager, M. A., Diehm, C., ... American Heart Association Council on Peripheral Vascular Disease., Council on Epidemiology and Prevention., Council on Clinical Cardiology., Council on Cardiovascular Nursing., Council on Cardiovascular Radiology and Intervention, and Council on Cardiovascular Surgery and Anesthesia. (2012). Measurement and interpretation of the ankle-brachial index a scientific statement from the American Heart Association. Circulation, 126(24), 2890-2909.

Adair, L. S. (2004). Dramatic rise in overweight and obesity in adult Filipino women and risk of hypertension. Obesity Research, 12(8), 1335-1341.

Adair, L. S., Kuzawa, C., McDade, T., Carba, D. B., \& Borja, J. B. (2018). Seventeen-year changes in body mass index, waist circumference, elevated blood pressure, and diabetes phenotypes in a cohort of Filipino women. Asia-Pacific Journal of Public Health, 30(6), 561-571.

Adair, L. S., Popkin, B. M., Akin, J. S., Guilkey, D. K., Gultiano, S., Borja, J., ... Hindin, M. J. (2011). Cohort profile: The Cebu longitudinal health and nutrition survey. International Journal of Epidemiology, 40(3), 619-625.

Alberti, G., Zimmet, P., Shaw, J., \& Grundy, S. M. (2006). The IDF consensus worldwide definition of the metabolic syndrome. Brussels: International Diabetes Federation, 23(5), 469-480.

Caro, J., Migliaccio-Walle, K., Ishak, K. J., \& Proskorovsky, I. (2005). The morbidity and mortality following a diagnosis of peripheral arterial disease: Longterm follow-up of a large database. BMC Cardiovascular Disorders, 5, 14.

Criqui, M. H., \& Aboyans, V. (2015). Epidemiology of peripheral artery disease. Circulation Research, 116(9), 1509-1526.

Criqui, M. H., Langer, R. D., Fronek, A., Feigelson, H. S., Klauber, M. R., McCann, T. J., \& Browner, D. (1992). Mortality over a period of 10 years in patients with peripheral arterial disease. The New England Journal of Medicine, 326(6), 381-386.

Ferreira, C. E., Franca, C. N., Correr, C. J., Zucker, M. L., Andriolo, A., \& Scartezini, M. (2015). Clinical correlation between a point-of-care testing system and laboratory automation for lipid profile. Clinica Chimica Acta, 446, 263-266.

FNRI-DOST. 2015a. Philippine Nutrition Facts and Figures 2013: 8th National Nutrition Survey: Dietary Survey. Manila: Food and Nutrition Research Institute of the Department of Science and Technology (FNRI-DOST) of the Philippines.

FNRI-DOST. 2015b. Philippine Nutrition Facts and Figures 2013: 8th National Nutrition Survey: Clinical and Health Survey. Manila: Food and Nutrition Research Institute-Department of Science and Technology (FNRI-DOST) of the Philippines.

Fowkes, F. G., Murray, G. D., \& Butcher, I. (2008). Ankle brachial index combined with Framingham risk score to predict cardiovascular events and mortality. JAMA, 300(2), 197-208.

Fowkes, F. G. R., Aboyans, V., Fowkes, F. J. I., McDermott, M. M., Sampson, U. K. A., \& Criqui, M. H. (2016). Peripheral artery disease: Epidemiology and global perspectives. Nature Reviews Cardiology, 14(3), 156-170.

Gurven, M., Kaplan, H., Winking, J., Eid Rodriguez, D., Vasunilashorn, S., Kim, J. K., ... Crimmins, E. (2009). Inflammation and infection do not promote arterial aging and cardiovascular disease risk factors among lean horticulturalists. PLoS One, 4(8), e6590.
Hajer, G. R., Haeften, V., W, T., \& Visseren, F. L. J. (2008). Adipose tissue dysfunction in obesity, diabetes, and vascular diseases. European Heart Journal, 29(24), 2959-2971.

He, Y., Jiang, Y., Wang, J., Fan, L., Li, X., \& Hu, F. B. (2006). Prevalence of peripheral arterial disease and its association with smoking in a populationbased study in Beijing, China. Journal of Vascular Surgery, 44(2), 333-338.

Hunter, G. R., McCarthy, J. P., \& Bamman, M. M. (2004). Effects of resistance training on older adults. Sports Medicine, 34(5), 329-348.

International Expert Committee. (2009). International expert committee report on the role of the A1C assay in the diagnosis of diabetes. Diabetes Care, 32(7), 1327-1334.

Ix, J. H., Biggs, M. L., Kizer, J. R., Mukamal, K. J., Djousse, L., Zieman, S. J., et al. (2011). Association of body mass index with peripheral arterial disease in older adults the cardiovascular health study. American Journal of Epidemiology, 174(9), 1036-1043.

Johnston, L. E., Stewart, B. T., Yangni-Angate, H., Veller, M., Upchurch, G. R., Gyedu, A., \& Kushner, A. L. (2016). Peripheral arterial disease in subSaharan Africa: A review. JAMA Surgery, 151(6), 564-572.

Lohman, T. G., Roche, A. F., \& Martorell, R. (1991). Anthropometric standardization reference manual. Champaign, IL: Human Kinetics Books 90 pp.

Lozano, R., Naghavi, M., Foreman, K., Lim, S., Shibuya, K., Aboyans, V., ... Murray, C. J. L. (2012). Global and regional mortality from 235 causes of death for 20 age groups in 1990 and 2010: A systematic analysis for the global burden of disease study 2010. The Lancet, 380(9859), 2095-2128.

Mathers, C. D., \& Loncar, D. (2006). Projections of global mortality and burden of disease from 2002 to 2030. PLoS Medicine, 3(11), e442.

McDade, T. W., Borja, J. B., Kuzawa, C. W., Perez, T. L., \& Adair, L. S. (2015). C-reactive protein response to influenza vaccination as a model of mild inflammatory stimulation in The Philippines. Vaccine, 33(17), 2004-2008.

McDade, T. W., Burhop, J., \& Dohnal, J. (2004). High-sensitivity enzyme immunoassay for C-reactive protein in dried blood spots. Clinical Chemistry, 50 (3), 652-654.

McDade, T. W., Rutherford, J., Adair, L., \& Kuzawa, C. W. (2010). Early origins of inflammation: Microbial exposures in infancy predict lower levels of C-reactive protein in adulthood. Proceedings of the Biological Sciences, 277 (1684), 1129-1137.

McDade, T. W., Williams, S., \& Snodgrass, J. J. (2007). What a drop can do: Dried blood spots as a minimally invasive method for integrating biomarkers into population-based research. Demography, 44(4), 899-925.

Newman, A. B., Siscovick, D. S., Manolio, T. A., Polak, J., Fried, L. P., Borhani, N. O., \& Wolfson, S. K. (1993). Ankle-arm index as a marker of atherosclerosis in the cardiovascular health study. Cardiovascular heart study (CHS) collaborative research group. Circulation, 88(3), 837-845.

Norris, S. A., Osmond, C., Gigante, D., Kuzawa, C. W., Ramakrishnan, L., Lee, N. R., ... the COHORTS Group. (2012). Size at birth, weight gain in infancy and childhood, and adult diabetes risk in five low- or middle-income country birth cohorts. Diabetes Care, 35(1), 72-79.

Ochi, M., Kohara, K., Tabara, Y., Kido, T., Uetani, E., Ochi, N., ... Miki, T. (2010). Arterial stiffness is associated with low thigh muscle mass in middleaged to elderly men. Atherosclerosis, 212(1), 327-332.

Pearson, T. A., Mensah, G. A., Alexander, R. W., Anderson, J. L., Cannon, R. O., 3rd, Criqui, M., et al. (2003). Markers of inflammation and cardiovascular disease: Application to clinical and public health practice: A statement for healthcare professionals from the Centers for Disease Control and Prevention and the American Heart Association. Circulation, 107(3), 499-511.

Pedro, M., Barba, C., \& Leon, R. (2007). Nutrition transition in The Philippines. Philippines Population Review, 6(1), 1-19.

Popkin, B. M. (2001). Nutrition in transition: The changing global nutrition challenge. Asia Pacific Journal of Clinical Nutrition, 10(s1), S13-S18.

Resnick, H. E. (2004). Relationship of high and low ankle brachial index to allcause and cardiovascular disease mortality: The strong heart study. Circulation, 109(6), 733-739.

Roth, G. A., Forouzanfar, M. H., Moran, A. E., Barber, R., Nguyen, G., Feigin, V. L., ... Murray, C. J. L. (2015). Demographic and epidemiologic drivers of global cardiovascular mortality. New England Journal of Medicine, 372(14), 1333-1341.

Selvin, E. (2004). Prevalence of and risk factors for peripheral arterial disease in the United States: Results from the National Health and nutrition examination survey, 1999-2000. Circulation, 110(6), 738-743. 
Srikanthan, P., \& Karlamangla, A. S. (2011). Relative muscle mass is inversely associated with insulin resistance and prediabetes. Findings from the third National Health and nutrition examination survey. The Journal of Clinical Endocrinology and Metabolism, 96(9), 2898-2903.

Sritara, P., Sritara, C., Woodward, M., Wangsuphachart, S., Barzi, F., Hengprasith, B., \& Yipintsoi, T. (2007). Prevalence and risk factors of peripheral arterial disease in a selected Thai population. Angiology, 58(5), 572-578.

Visser, M., Pahor, M., Taaffe, D. R., Goodpaster, B. H., Simonsick, E. M., Newman, A. B., ... Harris, T. B. (2002). Relationship of interleukin-6 and tumor necrosis factor-alpha with muscle mass and muscle strength in elderly men and women: The health ABC study. The Journals of Gerontology. Series A, Biological Sciences and Medical Sciences, 57(5), M326-M332.

Wexler, L., Brundage, B., Crouse, J., Detrano, R., Fuster, V., Maddahi, J., ... Taubert, K. (1996). Coronary artery calcification: Pathophysiology, epidemiology, imaging methods, and clinical implications: A statement for health professionals from the American Heart Association. Circulation, 94(5), 1175-1192.
Woo, J., Lynn, H., Wong, S. Y. S., Hong, A., Tang, Y. N., Lau, W. Y., ... Kwok, T. C. Y. (2006). Correlates for a low ankle-brachial index in elderly Chinese. Atherosclerosis, 186(2), 360-366.

World Bank. 2018. The World Bank in the Philippines. URL:http://www. worldbank.org/en/country/philippines/overview.

How to cite this article: Kuzawa CW, Barrett TM, Borja JB, et al. Ankle brachial index (ABI) in a cohort of older women in the Philippines: Prevalence of peripheral artery disease and predictors of ABI. Am J Hum Biol. 2019;31:e23237. https://doi.org/10.1002/ ajhb.23237 